\title{
Better IVF outcomes following improvements in laboratory air quality
}

\author{
Rabea Youcef Khoudja • Yanwen Xu • Tao Li • \\ Canquan Zhou
}

Received: 19 September 2012 / Accepted: 19 November 2012 /Published online: 16 December 2012

(C) The Author(s) 2012. This article is published with open access at Springerlink.com

\begin{abstract}
Background It has been proved that air quality is crucial for the success of IVF because of the presence of volatile organic compounds (VOCs), microbes, and perfumes, all of which can be harmful to embryo development in vitro. Therefore IVF laboratories are equipped with high efficiency particulate air (HEPA), and activated carbon filters plus positive pressure for air particulate control, with or without CODA system. Here we introduce a new technology using specially treated Honeycomb matrix media aligned in the Landson ${ }^{\mathrm{TM}}$ series system for our laboratory air purification and its impact on IVF outcome. Methods Air samples were collected outside and inside the laboratory, and intra-incubator at three different time points, before and after changing carbon filters and after Landson system installation, and we correlated air compounds measure variation with IVF outcome from 1403 cycles.

Results An improvement of air quality was confirmed with passages of total VOCs from $0.42 \mathrm{mg} / \mathrm{m}^{3}, 30.48 \mathrm{mg} / \mathrm{m}^{3}$, $9.62 \mathrm{mg} / \mathrm{m} 3$, to $0.1 \mathrm{mg} / \mathrm{m}^{3}, 2.5 \mathrm{mg} / \mathrm{m}^{3}, 2.19 \mathrm{mg} / \mathrm{m}^{3}$ through $0.07 \mathrm{mg} / \mathrm{m}^{3}, 0.16 \mathrm{mg} / \mathrm{m}^{3}, 0.29 \mathrm{mg} / \mathrm{m}^{3}$, outside the laboratory, inside laboratory and intra-incubator respectively at three separated air sampling times. A clear decrease was observed in some VOCs such as formaldehyde, ethylene, acethylene, propylene, SO2, pentane, NOx, benzene, Hallon-1211, CFC and alcohol.

At the same time a significant difference $(P<0.05)$ was found between the third testing time TT3 after carbon filter
\end{abstract}

Capsule We developed a novel air purification method utilizing specially treated honeycomb matrix media with a LandsonTM system, which significantly improved IVF laboratory air quality, and resulted in better embryo quality, and higher pregnancy and implantation rates.

R. Y. Khoudja $\cdot$ Y. Xu $・$ T. Li $\cdot$ C. Zhou $(\bowtie)$

Reproductive Medicine Center, The First Affiliated Hospital of

SUN Yat-sen University, 58 Zhongshan Road II,

Guangzhou, China 510080

e-mail: zhoucanquan@gmail.com change and Landson system installation and the first testing time TT1 before carbon filter change in fertilization rate $83.7 \%$ vs $70.1 \%$, embryo cleavage rate $97.35 \%$ vs $90.8 \%$, day 5 blastocyst formation rate $51.1 \%$ vs $41.7 \%$, and pregnancy/implantation rates $54.6 \%, 34.4 \%$ vs $40.6 \%, 26.4 \%$. Conclusion Air purification by the new technology of Landson ${ }^{\mathrm{TM}}$ series significantly improved IVF laboratory air quality, and embryo quality, thus increased pregnancy and implantation rates.

Keywords Air quality control $\cdot$ VOCs $\cdot$ IVF laboratory $\cdot$ IVF outcome $\cdot$ Embryo $\cdot$ Pregnancy rate $\cdot$ Implantation rate

\section{Introduction}

Creating an optimal environment for embryo culture is important for ensuring embryo viability, and thereby maintaining stable pregnancy outcome. Various factors, such as air quality, temperature, and light, are known to affect oocytes and embryos. Air quality, in particular, is easy to overlook when pregnancy rates start to decline. Volatile organic compounds (VOCs) are very harmful to embryos [1]. During embryonic growth and development, VOCs directly attach to DNA and abort growth [2-4] found that episodic air pollution is associated with increased DNA fragmentation in human sperm without other changes in semen quality. Many studies have also documented that small amounts of VOCs in the circulating air of an IVF laboratory can have detrimental effects on pregnancy rates $[5,6]$.

It has been well documented that the ambient air of IVF laboratories carries harmful VOCs (e.g. styrenes, formaldehydes, glutealdehydes, toluene, etc.), malignant microbes, perfumes, deodorants, and even odors from the outside environment, which affect embryonic development [7-9]. Air contaminants, such as chemical air contaminants (CACs) 
and VOCs, which are introduced from various sources, may interact with samples, tissues, media, and oil, and consequently, have serious effects on IVF outcome $[5,10,11,12]$. Thus, it is essential to set-up an air filtration/purification system that is efficient, quiet, affordable, and has the ability to filter hydrocarbon pollutants, VOCs, and chemically active compounds, and thereby eliminate airborne pathogens $[13,14]$.

Due to the growing amount of evidence suggesting that air contaminants can affect IVF outcome, cleanroom specifications for particulate and microorganism contamination in IVF laboratories have been enforced by European Union laws under Directive 2006/86/EC [15]. However, the removal of these contaminants would require very advanced air handling systems [16]. High efficiency particulate air (HEPA) and activated carbon filters, positive pressure, and general sterility precautions can prevent contamination. Specifically, the outside air brought into the unit is first filtered with activated carbon, which removes various hydrocarbons, and then HEPA removes the particulate materials (0.3microns). Furthermore, the carbonactivated air filtration (CODA) system was introduced in 1997. It consists of a CODA incubator filtration unit, which is used within the incubator and environmental chamber, a CODA $\mathrm{CO}_{2}$ and Tri-Gas inline filter, which is used as the incoming gas lines, and the CODA tower, which filters the air in the laboratory, procedure rooms, and working environment. Numerous studies have demonstrated improvements in pregnancy and implantation rates after using the CODA system $[17,18]$.

Recently, we applied a novel air purification technology in our laboratory to remove airborne molecular contaminants (AMCs), chemical air contaminants (CACs) and volatile organic compounds (VOCs) in order to improve the air quality in our laboratory, using a specially treated honeycomb matrix media (MeadWestvaco Corporation) aligned in Landson ${ }^{\mathrm{TM}}$ series system (Sinolandy. Technology Company); which is commercialized for IVF laboratory air filtration equipment applying an optimized air dynamic and potential chemical catalytic mechanism for air intake, pre-filter section, MeadWestvaco honeycomb File, power system, and post-filter protection, and an inline gas separator to purify the intralaboratory air, and was installed inside our laboratory.

Thus, the aim of the present study was to assess the impact of this novel technology on laboratory air quality by VOC concentration changes, as well as embryogenesis parameters (i.e. fertilization, cleavage, and embryo quality), and pregnancy and implantation rates, before and after the installation of the novel air filtration system.

\section{Materials and methods}

Our laboratory is approximately $130 \mathrm{~m}^{3}$, and was previously equipped with a HEPA air filtration system with activated carbon filters, one Gen X CODA tower laboratory air cleaner, two Low-Boy CODA, one CODA Aero, and CODA filters in some incubators, which were changed every 3 6 months. On August 10th, 2011 we installed a new intralaboratory air filtration system (i.e. Landson ${ }^{\mathrm{TM}}$ system) while maintaining the previous components. The last change in carbon filters was on August 2010.

Following the changes to the air filtration system, we assessed various embryogenesis parameters (i.e. fertilization, day 3 embryos cleavage, blastocyst formation, pregnancy, and embryo implantation rates) in our laboratory at three separate testing times (TT). They were as follows: i) TT1 was between April 1 and May 25, 2011, which was prior to the carbon filter change, and thus the first air sample was collected on May 25th; ii) TT2 was between May 25 and August 10, 2011, which was after the carbon filter change (i.e. May 25), but prior to the installation of the new Landson ${ }^{\mathrm{TM}}$ system, and thus, the second air sample was collected on June 10th; and TT3 was between August 11 and October 10, 2011, which was after the installation of the Landson ${ }^{\mathrm{TM}}$ system on August 10th, and thus, the third air sample was collected on August 18th. Furthermore, these air samples were collected from three different places, namely the air in the hallway outside of the laboratory, which is supplied by an unfiltered air system, the air in the laboratory, and the air inside the same incubator. The air sampling time was $20-30 \mathrm{~min}$ in duration, and $100 \mathrm{~L}$ of air were collected into a Tedlar bag for analysis. using high performance liquid chromatography (HPLC) for aldehydes, gas-chromatography/mass spectrometry with an EnTech cryoconcentration system for VOCs, and GE online analyzer for nitrogen oxides (NOx) and sulfur dioxide (SO2).

\section{Patients}

Patients that underwent consecutive standard in vitro fertilization (IVF) and intra cytoplasmic sperm injection (ICSI) cycles at the Reproductive Medicine Center of the First Affiliated Hospital, Sun Yat-Sen University between April 1and October 10, 2011 were included in the study, and divided into three groups, according to the three testing times. The inclusion criteria were as follows: $<38$ years old, $\geq 4$ oocytes retrieved, less than 3 IVF cycles, and underwent a gonadotropin-releasing hormone agonists (GnRH-a) long protocol.

Assessment of fertilization and embryo quality

Embryogenesis parameters, as well as normal fertilization, cleavage, blastocyst formation, pregnancy, and implantation rates, were compared between the three groups (i.e. testing time). Normal fertilization was characterized by two visible and distinct pronuclei and two polar bodies. The day 3 (D3) embryo grade score was evaluated on the third day after 
oocyte recovery, based on the modified criteria of Ziebe et al. [19]. One to three embryos were transferred on day 3 or 5 , depending on the age and cycle number, and good quality supernumerary embryos were cryopreserved.

\section{Pregnancy testing}

Biochemical pregnancies were defined as a positive with a urine $\beta$ human chorionic gonadotrophin ( $\beta$-HCG) pregnancy test 18 days after follicle aspiration. Clinical pregnancies were confirmed by ultrasound at 7-8 weeks of gestation, and pregnancies were considered to be ongoing when there was at least one fetus with a vital heartbeat after 12 weeks of gestation.

\section{Statistical analysis}

Baseline characteristics (continuous data) are presented as mean and standard deviation (mean $\pm \mathrm{SD}$ ). Categorical variables are presented as absolute counts and percentages. Differences in fertilization, embryo cleavage, blastocyst formation, pregnancy, and implantation rates were determined via Pearson's chi-squared test and a Yates' continuity correction or a Fisher's exact test followed by a Bonferroni multiple comparisons test, where appropriate. A logistic regression analysis was performed to determine the influence of potential confounding variables (i.e. baseline characteristics) on the primary outcome. $P<0.05$ was considered as statistically significant. Statistical analyses were conducted with the SPSS statistical package (version 19.0) for Windows (IBM.SPSS Inc., USA).

\section{Results}

Air contaminants

There was a total of 49 compounds detected, which included one formaldehyde, two acids, 37 hydrocarbons and aromatics, and nine halogens. The total amounts of VOCs measured in the first air sample obtained from the hallway, laboratory, and incubators were $0.42,30.48$, and $9.62 \mathrm{mg} /$ $\mathrm{m}^{3}$, respectively. These levels declined to $0.10,2.50$, and $2.19 \mathrm{mg} / \mathrm{m}^{3}$, respectively, in the second air sample obtained 16 days after the activated carbon filters were changed, and further decreased to $0.07,0.16$, and $0.29 \mathrm{mg} / \mathrm{m}^{3}$, respectively, in the third air sample obtained 10 days after the installation of the Landson ${ }^{\mathrm{TM}}$ system. The VOCs that decreased significantly from the first to the third sampling time point are presented in Table 1 There were no or minimal changes observed in propylene, propane, heptane, xylene, cyclopentane, ethylbenzene, styrene and other molecule amounts.
Clinical assessments

Of the 1403 patients that were included in the study, 1188 received a fresh embryo transfer, the relevant demographic data, including age, infertility time, clinical data, oocytes retrieved, and enbryos transferred, are presented in table 2. Using logistic regression analysis, it was found that there were no significant correlations between the various potential confounding variables (i.e. age, baseline hormone levels, stimulation protocol, total dose of gonadotropin, days of stimulation, mean oocytes retrieved, and mean embryos transferred).

Table 3 presents the total fertilization rates, normal fertilization rates, cleavage rates, blastocyst formation rates, abortion rates, pregnancy rates, and implantation rates of the three groups. Fertilization rates at TT3 and TT2 were significantly higher than at TT1 $(83.7$ and $82.3 \%$ vs. $70.1 \%$ ), and there were no significant differences found between TT2 and TT3. The normal fertilization rate at TT3 was significantly higher than both TT2 and TT1 (66.3\% vs. 64.6 and $61.3 \% ; P<0.05)$. There was also a significant difference between TT1 and TT2 $(P<0.016)$. D3 embryo cleavage rate was calculated for the all normal fertilized embryos (2pronucleus: $2 \mathrm{PN}$ ), and it was found that there was a significant difference between TT3 cleaved embryos and those of TT1 and TT2 (97.3\% vs. 90.8 and $94.1 \%)$. There were also significant differences between TT1 and TT2 with respect to cleavage rate. Day 5 blastocyst formation rate had also evaluated at each testing time. At TT3, 499 blastocyts formed from 977 cultured embryos (51.1\%) compared to 41.7 and $38.1 \%$ at TT1 and TT2, respectively $(P<0.05)$. Furthermore, there was a decline in blastocyst formation rate noted at TT2 compared to TT1; however, it was not statistically significant.

A total of 173 pregnancies were successful from 317 fresh embryo transfers (54.6\%), and 248 gestational sacs were observed via ultrasound examination at 7 weeks after embryo transfer with an implantation rate of $34.4 \%$ (248/ $721)$ in the TT3 group, which was significantly higher than that of the TT1 group $(26.4 \% ; 240 / 910)$. There were no significant differences in pregnancy rates between TT2 and TT3, and implantation rates between TT1 and TT2 (Fig. 1). However, there were significant differences in pregnancy rates between TT1 and TT2 (29 vs. $46.7 \%$ ), and implantation rates between TT2 and TT3 (28.6 vs. $34.4 \%$ ). There were no significant differences between groups in regards to the abortion rate.

\section{Discussion}

VOCs are detrimental to the success of IVF laboratories. VOCs are hydrocarbon-based compounds that are emitted 
Table 1 level of some VOC in different areas, on different sampling time

\begin{tabular}{|c|c|c|c|c|c|c|c|c|c|}
\hline \multirow[t]{2}{*}{$\mathrm{VOC} \mu \mathrm{g} / \mathrm{m}^{3}$} & \multicolumn{3}{|c|}{ First sampling } & \multicolumn{3}{|c|}{ Second sampling } & \multicolumn{3}{|c|}{ Third sampling } \\
\hline & hallway & lab & Incubator & hallway & lab & Incubator & hallway & lab & Incubator \\
\hline Acetylene & 1.92 & 2.56 & 3.25 & 4.34 & 4.68 & 5.72 & 2.16 & 1.31 & 1.98 \\
\hline $\mathrm{SO}_{2}$ & 0.96 & 1.11 & 1.42 & 1.42 & 1.07 & 0.79 & 0.71 & 0.29 & 0.46 \\
\hline Pentane & 2.87 & 3.56 & 3.8 & 6.91 & 5.86 & 5.5 & 0.9 & 1.48 & 1.6 \\
\hline NOx & 17.29 & 12.72 & 12.98 & 11.96 & 6.8 & 7.4 & 10.6 & 6.28 & 7.0 \\
\hline Benzene & 6.36 & 5.12 & 7.17 & 2.8 & 2.3 & 2.1 & 0.87 & 1.35 & 1.76 \\
\hline Halon-1211 & 303 & 24609 & 9390 & 26 & 2317 & 1986 & 23 & 51 & 113 \\
\hline CFC11 & 2.13 & 2.75 & 2.26 & 1.47 & 1.45 & 1.4 & 0.5 & 0.6 & 0.8 \\
\hline $\mathrm{CFC} 12$ & 3.27 & 5.36 & 4.09 & 4.46 & 3.86 & 3.7 & 1.32 & 1.43 & 1.23 \\
\hline $\mathrm{CFC} 13$ & 0.97 & 1.16 & 1.1 & 0.73 & 1.3 & 0.65 & 0.26 & 0.28 & 0.36 \\
\hline Alcohol & 59.63 & 5792 & 140 & 3.97 & 141.8 & 119.86 & 8.79 & 73.57 & 79.22 \\
\hline Formaldehyde & 13.05 & 13.47 & 8.58 & 13.68 & 10.7 & 9.68 & 6.35 & 8.78 & 9.54 \\
\hline Ethylene & 0.07 & 0.56 & 1.15 & 0.75 & 0.83 & 6.46 & 0.24 & 1.1 & 4.62 \\
\hline Acethylene & 1.92 & 2.56 & 3.25 & 4.34 & 4.86 & 5.72 & 2.16 & 1.31 & 1.98 \\
\hline Ethane & 0.39 & 0.96 & 2.96 & 1.09 & 0.82 & 0.15 & 0.31 & 0.15 & 1.14 \\
\hline Propylene & 0.53 & 1.42 & 7.78 & 1.45 & 1.37 & 7.93 & 0.34 & 0.6 & 18.74 \\
\hline Isobutene & 6.32 & 8.96 & 9.99 & 10.19 & 6.58 & 6.4 & 1.22 & 1.58 & 3.01 \\
\hline Cis-butene & 0.13 & 0.27 & 0.85 & 0.6 & 0.5 & 0.56 & 0.19 & 0.14 & 0.12 \\
\hline Cyclopentane & 0.37 & 1.52 & 1.56 & 0.22 & 0.73 & 0.40 & 0.3 & 0.25 & 0.36 \\
\hline
\end{tabular}

Table 2 Patient baseline characteristics and cycle parameters

\begin{tabular}{|c|c|c|c|}
\hline Item $($ mean $\pm \mathrm{SD})$ & TT1 & TT2 & TT3 \\
\hline Patients included (n) & 446 & 573 & 384 \\
\hline Routine IVF cycles & 277 & 310 & 193 \\
\hline ICSI cycles & 144 & 230 & 160 \\
\hline Half-ICSI & 25 & 33 & 31 \\
\hline Total ET & 396 & 475 & 317 \\
\hline D3 ET & 383 & 420 & 281 \\
\hline D5 ET & 13 & 55 & 36 \\
\hline Female age (years) & $30.5 \pm 3.4(22-37)$ & $30.3 \pm 3.4(20-37)$ & $30.5 \pm 3.8(21-37)$ \\
\hline Infertility time (years) & $4.4 \pm 2.8$ & $4.4 \pm 2.7$ & $4.3 \pm 2.9$ \\
\hline Days of stimulation & $10.3 \pm 1.8$ & $10.6 \pm 4.6$ & $11.2 \pm 1.9$ \\
\hline Total GnRH used (IU) & $2117 \pm 640$ & $2059 \pm 640$ & $2185 \pm 761$ \\
\hline Basal-FSH (IU/ml) & $5.7 \pm 1.7$ & $5.7 \pm 1.4$ & $5.7 \pm 1.4$ \\
\hline Basal-LH (IU/ml) & $3.5 \pm 2.5$ & $3.4 \pm 1.9$ & $3.4 \pm 1.7$ \\
\hline Basal-E2 (pg/ml) & $35.4 \pm 17.4$ & $32.8 \pm 14.8$ & $34.2 \pm 15.6$ \\
\hline Basal-T (nmol/l) & $0.7 \pm 0.4$ & $0.6 \pm 0.4$ & $0.6 \pm 0.3$ \\
\hline HCG day FSH (IU/ml) & $13.7 \pm 4.6$ & $12.8 \pm 4.8$ & $12.7 \pm 5.6$ \\
\hline HCG day E2 (pg/ml) & $2924 \pm 1293$ & $3154 \pm 1224$ & $3308 \pm 1341$ \\
\hline HCG day LH (IU/ml) & $0.85 \pm 0.6$ & $0.92 \pm 2.1$ & $1.1 \pm 3.2$ \\
\hline HCG day P (ng/ml) & $0.6 \pm 0.3$ & $0.6 \pm 0.4$ & $0.6 \pm 0.4$ \\
\hline Mature follicle $(>18 \mathrm{~mm})$ on HCG day & $6.1 \pm 2.8$ & $6.9 \pm 2.7$ & $7.5 \pm 2.4$ \\
\hline HCG day endometrial thickness (mm) & $11.3 \pm 2.4$ & $11.3 \pm 2.6$ & $11.4 \pm 2.4$ \\
\hline No of retrieved oocytes & 6186 & 8210 & 5953 \\
\hline Mean no. of oocytes & $13.9 \pm 7.4$ & $14.5 \pm 7.5$ & $15.6 \pm 7.6$ \\
\hline Transferred embryos & 910 & 1053 & 721 \\
\hline Mean of transferred embryos & $2.3 \pm 0.5$ & $2.2 \pm 0.5$ & $2.2 \pm 0.5$ \\
\hline
\end{tabular}


Table 3 Embryogenesis parameters and IVF outcomes measures

\begin{tabular}{|c|c|c|c|c|}
\hline Item & TT1 & TT2 & TT3 & $P$ value \\
\hline OPU cycle & 446 & 573 & 384 & \\
\hline ET number & 396 & 473 & 317 & \\
\hline Retrieved oocytes & 6186 & 8210 & 5953 & \\
\hline ET embryos & 910 & 1053 & 721 & \\
\hline Fertilization rate $^{\mathrm{a}}(\%)$ & $70.1 \%(4336 / 6186)$ & $82.3 \%(6759 / 8210)$ & $83.7 \%(4984 / 5953)$ & $<0.001^{\mathrm{c}, \mathrm{d}}$ \\
\hline Normal fertilisation $^{\mathrm{a}}(2 \mathrm{PN})(\%)$ & $61.3 \%(3791 / 6186)$ & $64.6 \%(5303 / 8210)$ & $66.3 \%(3931 / 5953)$ & $<0.001^{\mathrm{c}}$ \\
\hline Cleavage rate ${ }^{\mathrm{b}}$ & $90.8 \%(3441 / 3791)$ & $94.15 \%(4993 / 5303)$ & $97.35 \%(3827 / 3931)$ & $<0.001^{\mathrm{c}}$ \\
\hline Blastocyst culture & 568 & 1084 & 977 & \\
\hline Blastocyst formation rate & $41.7 \%(237 / 568)$ & $38.1 \%(413 / 1084)$ & $51.07 \%(499 / 977)$ & $<0.001^{\mathrm{c}, \mathrm{d}}$ \\
\hline Pregnancy rate (PR) & $40.6 \%(161 / 396)$ & $46.7 \%(222 / 475)$ & $54.6 \%(173 / 317)$ & $0.001^{\mathrm{c}, \mathrm{d}, \mathrm{e}}$ \\
\hline Implantation rate (IR) & $26.4 \%(240 / 910)$ & $28.6 \%(301 / 1053)$ & $34.4 \%(248 / 721)$ & $0.001^{\mathrm{c}, \mathrm{d}, \mathrm{e}}$ \\
\hline Abortion rate & $4.9 \%(8 / 161)$ & $6.7 \%(15 / 222)$ & $4.1 \%(13 / 317)$ & $0.38^{\mathrm{f}}$ \\
\hline
\end{tabular}

${ }^{a}$ Percentages, expressed per inseminated oocyte.

${ }^{\mathrm{b}}$ Percentage, expressed per $2 \mathrm{PN}$ fertilized oocyte

${ }^{\mathrm{c}}$ significantly different $P<0.001$ compared with $\mathrm{d}$ and e

${ }^{\mathrm{d}}$ No significant difference was found between: TT2 and TT3 on fertilization rate, PR and IR

${ }^{\mathrm{e}}$ No significant difference was found between: TT1 and TT2 on blastocyst formation rate, PR and IR $(P>0.016)$

${ }^{\mathrm{f}}$ No significant difference $P>0.05$

by various industries, vehicles, and heat exhausts, as well as by a variety of cleaning products, instruments, such as microscopes, television monitors, computers, and furniture. Additionally, due to their manufacturing process, perfumes, aftershave, and other highly scented aerosols may also release VOCs. Any new construction materials (e.g. paint) and furniture also release VOCs. New furniture made, in part, from particle board can emit VOCs, as it consists of $10 \%$ formaldehyde resin, which is capable of emitting gasses over 20 years. Consequently, unexpected sources of VOCs are commonly found in IVF laboratories. These can include cleaning agents, perfumes, cabinets, grease on the wheels of equipment, sources in heating ventilating and air conditioning (HVAC) equipment, and many stainless steel cabinets, which typically have a $3 / 4$ inch thick piece of particle board used under the steel to provide rigidity to the countertop [7-9].

High levels of VOCs (over $1 \mathrm{ppm}$ ) are directly toxic to embryos, as determined via murine and human experiments [20]. VOC levels around $0.5 \mathrm{ppm}$ will typically allow for acceptable blastocyst development and reasonable pregnancy rates, but unfortunately, there are a high percentage of miscarriages. Ideally, VOC levels should be below $0.2 \mathrm{ppm}$, but preferably zero. Cohen et al. [8] found that after moving their IVF laboratory, their pregnancy rates dropped significantly due to high levels of VOCs. They also had a drop in their pregnancy rates when a neighbor in the building replaced their vinyl floor, which requires the use of large
Fig. 1 IVF outcome before and after installation of air filtration new system

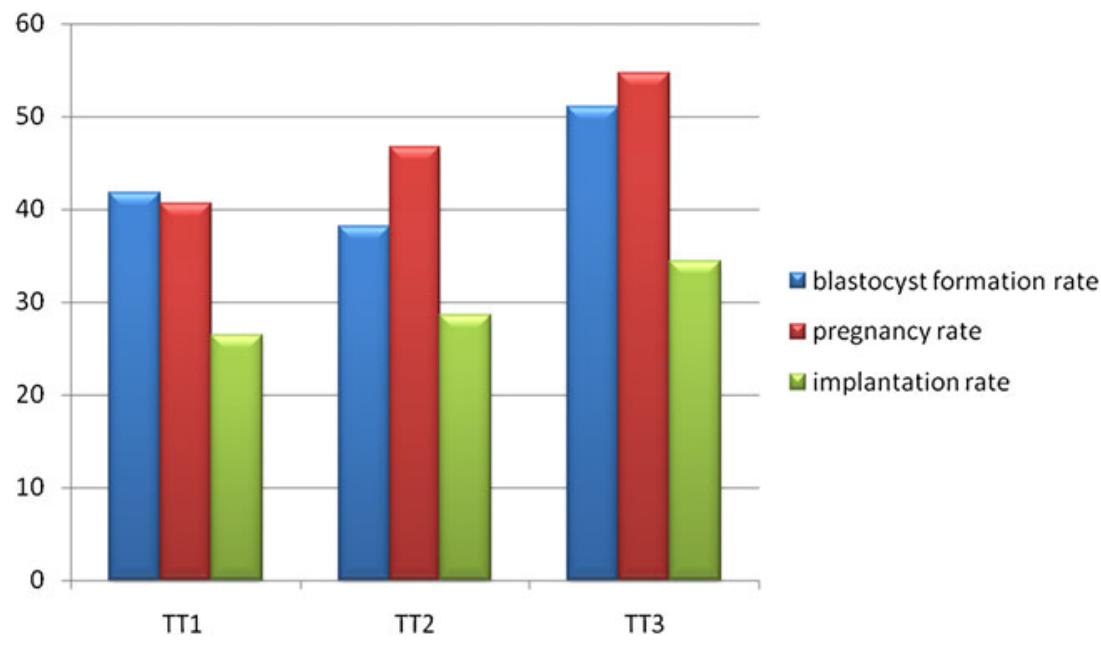


amounts of adhesive (i.e. a VOC source), and thereby contaminated their IVF laboratory. Hall et al. [9] published the mean VOC levels of various areas in seven IVF laboratories. The mean VOC levels were $0.53 \mathrm{mg} / \mathrm{m}^{3}$ in the outside air, $1.152 \mathrm{mg} / \mathrm{m}^{3}$ in the air supply, $2.862 \mathrm{mg} / \mathrm{m}^{3}$ inside the laboratory, $2.769 \mathrm{mg} / \mathrm{m}^{3}$ in the incubator, and $4.372 \mathrm{mg} /$ $\mathrm{m}^{3}$ in the hallway around the laboratory. Furthermore, the average lower limit of air quality ranged from 330 to $2240 \mu \mathrm{g} / \mathrm{m}^{3}\left( \pm 636 \mu \mathrm{g} / \mathrm{m}^{3}\right)$. Consequently, all of these IVF laboratories were equipped with HEPA filters, pretreatment with carbon filtration impregnated with potassium permanganate, and over-pressure for particle control.

A number of studies have shown improved pregnancy rates, but not embryo morphological quality, with the use of CODA air filtration systems in human IVF laboratories [17, 21]. In one study assessing cattle IVF [18], it was found there were no significant effects of the CODA system, as judged by the percent cleavage and embryo quality, stage, or development; however, the pregnancy rate was improved significantly following the transfer of both fresh and frozen/ thawed embryos. It was speculated that there was an improvement in the intrinsic quality of the embryo, which was not manifested in the morphology. Esteves et al. [22] used inline-HEPA and carbon filters located between the gas cylinders and incubators within the intra-incubator filtration unit, which resulted in high cleavage rates, more good quality embryos, higher pregnancy rates, and low spontaneous abortion rates compared to using only a HEPA system and CODA tower. Furthermore, Forman et al. [23] reported an improvement in air quality and a decrease in VOCs, particulates, and aldehyde, after using a HEPA system with high activity charcoal, a potassium permanganate filter, and CODA. Nevertheless, some studies reported no changes in embryo quality or implantation rate with CODA versus nonCODA systems [24].

In China, CODA systems have been applied in the laboratory environment for many years. However, in our laboratory, there were no improvements in pregnancy rates documented with the use of the CODA system. Due to the poor air quality, high VOC levels, and the hot and humid weather in Guangzhou, the filters may became saturated earlier than anticipated, and thereby certain VOCs may not be removed. We hypothesize that the summertime seasonal elevations in temperature and humidity may also initiate the desorption of trapped VOCs from the carbon filters installed in the laboratory air handling system, and reduce the adsorption efficacy of the system [25].

On August 10, 2011, a new air filtration system, consisting of specially treated honeycomb matrix media fitted into the Landson ${ }^{\mathrm{TM}}$ system, was installed in our laboratory. This novel technology made clear improvements in the air quality by reducing VOCs levels, as well as making better IVF outcome, as determined via embryo quality, and pregnancy and implantation rates. Total VOC levels decreased outside $\left(0.42,0.10\right.$, and $\left.0.07 \mathrm{mg} / \mathrm{m}^{3}\right)$ and inside the laboratory $\left(30.48,2.50,0.16 \mathrm{mg} / \mathrm{m}^{3}\right)$, and in the intra-incubator $(9.62$, 2.19 , and $0.29 \mathrm{mg} / \mathrm{m}^{3}$ ) at three separate air sampling times (i.e. before and after carbon filter changes, and after Landson $^{\mathrm{TM}}$ system installation). Interestingly, there were also improvements in the outside air quality expressed by the air in the hallway during the third sampling period (TT3) compared to TT1, which may be, in part, responsible for the improvements in laboratory air quality. Fluctuations in outside air VOC levels are very important. Studies have shown that VOC concentrations are 2-5 times higher in indoors than outdoor environment and 3-4 times higher in winter than summer [26]. This may be due to a number of factors, including a low rate of air exchange, the unfiltered outside air may be cleaner than the HEPA-filtered laboratory air or air obtained from incubators, and the accumulation of VOCs derived from adjacent spaces or specific laboratory products, all of which complicate the situation further and make it hard to determine the sources of contaminated air [8, 12]; [27].

Of the 49 compounds detected, certain compounds, such as formaldehyde, decreased significantly between the first and third sampling time points. Between TT1 and TT3, formaldehyde levels decreased outside and inside the laboratory, but slightly increased in the intra-incubator, but these levels were still lower than the aldehyde levels of $57.6 \mu \mathrm{g} /$ $\mathrm{m}^{3}$ found in the exterior air and $12 \pm 26.4 \mu \mathrm{g} / \mathrm{m}^{3}$ in the incubator, as reported by Hall et al. [9]. Thus, our new filtration system ameliorated aldehyde concentrations inside the laboratory, but not in the intra-incubator. Formaldehyde is one of the most common VOCs. It is an off-gas from wood products, such as plywood or particle board, and produced by paints, varnishes, floor finishes, and cigarette smoking $[28,29]$. Formaldehyde does not accumulate within the environment, as it is broken down within a few hours by sunlight or bacteria present in the soil or water. Thus, its main origins in an IVF laboratory are primarily internal, such as plastics, personnel, and furnishings.

A study on Finnish women working in laboratories at least 3 days a week found a significant correlation between spontaneous abortion and formaldehyde exposure. Another study on Chinese women found abnormal menstrual cycles in $70 \%$ of the women occupationally exposed to formaldehyde compared to only $17 \%$ in the control group. After an earthquake hit Sichuan, China, a large number of survivors were housed in trailers made from medium-density fiberboard, which emitted up to 5 times China's maximum allowable formaldehyde levels. In April, 2009, there were 100 miscarriages recorded in this community, which may have been linked to the high exposure levels of formaldehyde [30]. Furthermore, Hall et al. [9] found an inversely correlation between mouse embryo development and different 
acrolein (ubiquitous aldehyde) concentrations. This is not surprising, as both $[31,32]$ found that aldehyde is highly toxic and affects all molecular mechanisms involved in cell replication $[7,10]$.

In the present study, similar findings to formaldehyde were found with respect to ethylene, acetylene, ethane, propylene, $\mathrm{SO}_{2}$, NOx isobutene, cis-butene, cyclopentane, benzene, CFC-11, chloroform, carbon tetrachloride, halon1211, and alcohol (Table 1). Specially, there were improvements in intra-laboratory air contamination compared to the intra-incubators, where the accumulation of these VOCs was difficult to remove. Schimmel et al. [33]. Most of these VOCs are primarily from internal source. Cohen et al. [8] reported that benzene, toluene, cyclenes, and other hydrocarbons (hexane) are highly concentrated in incubators because of the items placed in them, especially plastics. Styrene comes from Petri dishes or other materials used in sterile cultures. Gilligan et al. [34] Benzene is derived from gas bottles, and its levels are elevated in incubators, but decline with increasing distances from them. These results coincide simultaneously with the significant improvements in our laboratory parameters (e.g. fertilization, cleavage, embryo quality, and blastocyst formation rates), and consequently increases in pregnancy and implantation rates. This may present concerns regarding the possible implications of these particles on in vitro embryonic development, especially given the evidence from studies on the detrimental effects of direct exposures of related VOCs in animal embryo cultures [35].

In a study conducted between 2000 and 2007 on 7403 cycles, Legro et al. [35] found that exposure to an increased level of air pollutants, especially nitrogen dioxide, has been associated with lower likelihoods of successful pregnancy among women undergoing IVF.

Furthermore, in our study, there was a decline in the blastocyst formation rate during TT2 compared to TT1 (i.e. before the carbon filter change). While this was not statistically significant, it coincided with the clear increase in certain VOCs (i.e. ethylene, acethylene, ethane, isopentane, N-pentane, and heptane) inside the incubators and laboratory between TT1 and TT3. Thus, this finding requires further investigation and follow-up.

However, it should be noted that the novel air filtration system did not appear to have any effects in reducing propylene, propane, heptane, xylene, cyclopentane, ethylbenzene, styrene, methylpentane, methylbutene, methylchlorhexane, chloromethane, methylcyclopentane, trichloroethylene, and tetrachloroethylene levels.

In conclusion, there were significant improvements in air quality with the Landson ${ }^{\mathrm{TM}}$ system, which coincided with better pregnancy outcome. Further research on the fundamental effects of air quality on embryo development is warranted to improve IVF outcome. Additionally, more sensitive and optimized methodology for detecting changes in air contaminants, such as $\mathrm{H}_{2} \mathrm{~S}$ and other compounds, are warranted to improve our understanding of pregnancy fluctuations due to changes in air quality.

Acknowledgements We would like to thank Mr. Zhao Feng from MeadWestvaco Corporation for his helpful suggestions and the discussions regarding this manuscript.

Financial disclosures This study was founded by the Key Laboratory of the Guangdong Province and the Natural Science Foundation of China (81100472).

Open Access This article is distributed under the terms of the Creative Commons Attribution License which permits any use, distribution, and reproduction in any medium, provided the original author(s) and the source are credited.

\section{References}

1. Ritz B, Wilhelm M. Ambient air pollution and adverse birth outcomes: methodologic issues in an emerging. Field Basic Clin Pharmacol Toxicol. 2008;102(2):182-90.

2. Dadvand P, Rankin J, Rushton S, Pless-Mulloli T. Association between maternal exposure to ambient air pollution and congenital heart disease: a register-based spatiotemporal analysis. Am J Epidemiol. 2011;173(2):171-82.

3. Meng Z, Zhang LZ. Chromosomal aberrations and sisterchromatid exchanges in lymphocytes of workers exposed to sulfur dioxide. Mutat Res. 1990;241(1):15-20.

4. Rubes J, Selevan SG, Evenson DP, Zudova D, Vozdova M, Zudova Z, et al. Episodic air pollution is associated with increased DNA fragmentation in human sperm without other changes in semen quality. Hum Reprod. 2005;20(10):2776-83.

5. Boone WR, Johnson JE, Locke A-J, Crane MM. Control of air quality in an assisted reproductive technology laboratory. Fertil Steril. 1999;71:150-4.

6. Dickey RP, Wortham JWE, Potts A. Effect of IVF laboratory air quality on pregnancy success. Fertil Steril. 2010;94 Suppl 4:S 151.

7. Brown SK. Chamber assessment of formaldehyde and VOC emissions from wood-based panels. Indoor Air. 1999;9:209-15.

8. Cohen J, Gilligan A, Esposito W, Schimmel T, Dale B. Ambient air and its potential effects on conception in vitro. Hum Reprod. 1997;12:1742-1749-9.

9. Hall J, Gilligan A, Schimmel T, Cecchi M, Cohen J. The origin, effects and control of air pollution in laboratories used for human embryo culture. Hum Reprod. 1998;13(Supp14):146-55.

10. Johnson JE, Boone WR, Bernard RS. The effects of volatile compounds (VC) on the outcome of in vitro mouse embryo culture. Fertil Steril 1993; Suppl 1:S98-9.

11. Lundgren B, Jonsson B, Ek-Olausson B. Materials emission of chemicals - PVC flooring materials. Indoor Air. 1999;9:202-8

12. De Bortoli M, Kephalopoulos S, Kirchner S, Schauenburg H, Vissers H. State-of-the-art in the measurement of volatile organic compounds emitted from building products: results of European interlaboratory comparison. Indoor Air. 1999;9:103-16.

13. Chang JC, Fortmann R, Roache N, Lao HC. Evaluation of lowVOC latex paints. Indoor Air. 1999;9:253-8.

14. Elder K, Dale B. In vitro fertilization. 2nd ed. Cambridge: Cambridge University Press; 2000. p. 310.

15. European Union "Commission Directive 2006/86/EC implementing Directive 2004/23/EC of the European Parliament and of the 
Council as regards traceability requiements, notification of serious adverse reactions and events and certain technical requirements for the coding, processing, preservastion, storage and distribution of human tissues and cells" Official Journal of the European Union L294/32. 2006. Accessed 24 Nov 2006.

16. Gianaroli L, Plachot M, van Kooij R, Al-Hasani S, Dawson K, DeVos A, et al. Guidelines for good practice in IVF laboratories. Hum Reprod. 2000;15:2241-6.

17. Mayer JF, Nehchiri F, Weedon VM, Jones EL, Kalin HL, Oehninger $\mathrm{SC}$, et al. Prospective randomized crossover analysis of the impact of an IVF incubator air filtration system (coda, GenX) on clinical pregnancy rates. Fertility and Sterility Suppl. 1999;1:S42-3.

18. Merton JS, Vermeulen ZL, Otter T, Mullaart E, de Ruigh L, Hasler JF. Carbon-activated gas filtration during in vitro culture increased pregnancy rate following transfer of in vitro-produced bovine embryos. Theriogenology. 2007;67:1233-8.

19. Ziebe S, Peterson K, Lindenberg S, Andersen AG, Gabrielsen A, Andersen NA. Embryo morphology or cleavage stage: How to select the best embryos for transfer after in-vitro fertilization. Hum Reprod. 1997;12(No.7):545-9. ISSN 0268-1161.

20. Panizzo R. Air pollution linked to lower IVF success. Progress Educational rust. http://www.bionews.org.uk/page_58675.asp 2010.

21. Racowsky C, Jackson KV, Nurredin A, Balint $\bar{C}$, Shen S, de los Santos MJ. Carbon-activated air filtration results in reduced spontaneous abortion rates following IVF. Proceeding of the Eleventh world congress on in vitro fertilization and human reproductive genetics; Sydney Australia 1999.

22. Esteves SC, Gomes AP, Verza Jr S. Control of air pollution in assisted reproductive technology laboratory and adjacent areas improves embryo formation, cleavage and pregnancy rates and decreases abortion rate: comparison between a class 100 (ISO 5) and a class 1.000 (ISO 6) cleanroom for micromanipulation and embryo culture. Fertil Steril. 2004;82 Suppl 2:S259-60.

23. Forman M, Polanski V, Horvath P, Gilligan A, Rieger D. Reductions in volatile organic compounds, aldehydes, and particulate air contaminants in an IVF laboratory by centralized and stand-alone air filtration systems. Fertil Steril. 2004;82(2):S324.

24. Battaglia DE, Khabani A, Rainer C, Moore DE. Prospective randomized trial of incubator CODA filtration units revealed no effect on outcome parameters for IVF. Fertil Steril. 2001;75 Suppl 1:S6.

25. Worrilow KC, Huynh TH, et al. A retrospective analysis: seasonal decline in implantation rates and its correlation with increased levels of volatile organic compounds. Fertil Steril. 2002;78(1):S39.

26. EPA: united states environmental protection agency: An introduction to indoor air quality (IAQ), Volatiles organic compounds (VOCs). http://www.epa.gov/iaq/voc.html

27. Wolkoff P. Trends in Europe to reduce the indoor air pollution of VOCs. Indor Air. 2003;13(Suppl: 6):5-11.

28. Holyoak GR, Wang S, Liu Y, Bunch TD. Toxic effects of ethylene oxide residues on bovine embryos in vitro. Toxicology. 1996;108 (1-2):33-8.

29. Sparks LE, Guo Z, Chang JC, Tichenor BA. Volatile organic compound emissions from latex paint - part 1 - chamber experiment and source, model development. Indoor Air. 1999;9:10-7.

30. Tang X, Bai Y, Duong A, Smith MT, Li L, Zhuang L. Formaldehyde in china: production, consumption, exposure levels, and health effects. Environmental International. 2009;35 (8):1210-24.

31. Little SA, Mirkes PE. Relationship of DNA damage and embryotoxicity induced by 4-hydroperoxydechosphamide in postimplantation rat embryos. Teratology. 1990;41:223-31.

32. Zitting A, Heinonen T. Decrease of reduced glutathione in isolated rat hepatocytes caused by acrolein acrylonitrile, and the thermal degradation products of styrene copolymers. Toxicology. 1980;17:333-51.

33. Schimmel T, Gilligan A, Garrisi GJ, Esposito B, Cecci M, Dale B, et al. Removal of volatile organic compounds from incubators used for gamete and embryo culture. Reprod Fertil. 1997;68 Suppl 1:S165.

34. Gilligan T, Schimmel T, Esposito Jr B, Cohen J. Release of volatile organic compounds such as styrene by sterile Petri dishes and flasks used for in-vitro fertilization. Fertil Steril. 1997;68 Suppl $1: S 52-3$.

35. Legro RS, Sauer MV, Mottla GL, Richter KS, Li X, Dodson WC, et al. Effect of air quality on assisted human reproduction. Hum Reprod. 2010;25(5):1317-27. 